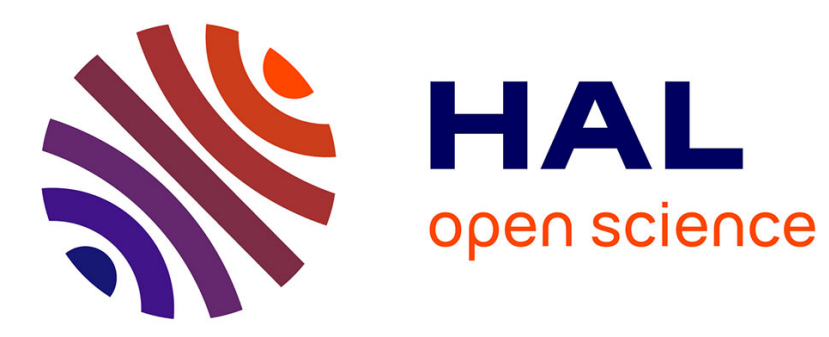

\title{
Steep nonlinear global modes in spatially developing media
}

\author{
Benoît Pier, Patrick Huerre, Jean-Marc Chomaz, Arnaud Couairon
}

\section{To cite this version:}

Benoît Pier, Patrick Huerre, Jean-Marc Chomaz, Arnaud Couairon. Steep nonlinear global modes in spatially developing media. Physics of Fluids, 1998, 10, pp.2433-2435. 10.1063/1.869784 . hal00119908

\section{HAL Id: hal-00119908 \\ https://hal.science/hal-00119908}

Submitted on 12 Dec 2006

HAL is a multi-disciplinary open access archive for the deposit and dissemination of scientific research documents, whether they are published or not. The documents may come from teaching and research institutions in France or abroad, or from public or private research centers.
L'archive ouverte pluridisciplinaire HAL, est destinée au dépôt et à la diffusion de documents scientifiques de niveau recherche, publiés ou non, émanant des établissements d'enseignement et de recherche français ou étrangers, des laboratoires publics ou privés. 


\title{
LETTERS
}

The purpose of this Letters section is to provide rapid dissemination of important new results in the fields regularly covered by Physics of Fluids. Results of extended research should not be presented as a series of letters in place of comprehensive articles. Letters cannot exceed three printed pages in length, including space allowed for title, figures, tables, references and an abstract limited to about 100 words. There is a three-month time limit, from date of receipt to acceptance, for processing Letter manuscripts. Authors must also submit a brief statement justifying rapid publication in the Letters section.

\section{Steep nonlinear global modes in spatially developing media}

\author{
Benoît Pier, ${ }^{\text {a) }}$ Patrick Huerre, Jean-Marc Chomaz, and Arnaud Couairon \\ Laboratoire d'Hydrodynamique (LadHyX), CNRS UMR 7646, Ecole polytechnique, \\ F-91128 Palaiseau cedex, France
}

(Received 16 April 1998; accepted 15 June 1998)

\begin{abstract}
A new frequency selection criterion valid in the fully nonlinear regime is presented for extended oscillating states in spatially developing media. The spatial structure and frequency of these modes are dominated by the existence of a sharp front connecting linear to nonlinear regions. A new type of fully nonlinear time harmonic solutions called steep global modes is identified in the context of the supercritical complex Ginzburg-Landau equation with slowly spatially varying coefficients. A similar formulation is likely to be applicable to fully nonlinear synchronized global oscillations in spatially developing free shear flows. (C) 1998 American Institute of Physics.
\end{abstract}

[S1070-6631(98)00410-3]

Spatially developing free shear flows such as mixing layers, ${ }^{1}$ wakes, ${ }^{2,3}$ and jets ${ }^{4}$ typically give rise to intrinsic selfsustained oscillations when they exhibit a sufficiently large region of absolute instability. ${ }^{5}$ Fluctuations saturate at a finite amplitude in the locally unstable regions of the flow and become tuned at an overall frequency. The intrinsic frequency and the associated spatial distribution of fluctuations define a global mode living on the underlying unstable basic flow. In the present letter we show the existence of fully nonlinear global modes with a sharp stationary front separating linear and nonlinear regions. The complex GinzburgLandau (CGL) equation is chosen as a model of open flows since families of linear and nonlinear wave solutions are readily determined analytically. As summarized below, the study of CGL models has been found to lead to linear frequency selection criteria $^{6}$ that remain applicable for the Navier-Stokes equations. ${ }^{7}$ The same approach is adopted here in the fully nonlinear context.

In the linear approximation, global frequency selection in doubly infinite domains is dictated by saddle point conditions ${ }^{6,7}$ imposed on the local linear dispersion relation. Such a criterion predicts remarkably well the vortex shedding frequency behind blunt edged plates. ${ }^{8}$ Nonlinear extensions of these concepts have only recently been developed, mainly in the context of various one-dimensional evolution models in semi-infinite ${ }^{9,10}$ or finite $^{11}$ domains. The results compare satisfactorily with numerical simulations and experiments for Taylor-Couette flow ${ }^{12}$ and Rayleigh-Bénard convection with throughflow. ${ }^{13,10}$ Surprisingly, fully nonlinear soft global modes of the CGL equation varying smoothly over a doubly infinite domain have been shown, ${ }^{14}$ by application of Wentzel-Kramers-Brillouin-Jeffreys (WKBJ) theory, to satisfy a nonlinear saddle point criterion which is formally analogous to its linear counterpart. Here we show the existence of a second class of nonlinear spatially extended states in doubly infinite domains: steep global modes with a sharp front.

We assume that the fluctuating complex scalar field $\psi(x, t)$ is governed by the supercritical CGL equation written as

$$
\begin{aligned}
i \frac{\partial \psi}{\partial t}= & \left(\omega_{0}(X)+\frac{1}{2} \omega_{k k}(X) k_{0}(X)^{2}\right) \psi+i \omega_{k k}(X) k_{0}(X) \frac{\partial \psi}{\partial x} \\
& -\frac{1}{2} \omega_{k k}(X) \frac{\partial^{2} \psi}{\partial x^{2}}+\gamma(X)|\psi|^{2} \psi,
\end{aligned}
$$

where the complex functions $\omega_{0}(X), \omega_{k k}(X), k_{0}(X)$, and $\gamma(X)$ solely depend on the slow space variable $X=\epsilon x$, $\epsilon \ll 1$, to account for the weak inhomogeneity of the medium. The choice of these functions and their meaning will become clear when we discuss the resulting linear and nonlinear dispersion relations.

In regions where the amplitude of $\psi$ is small, its behavior is governed by the linearized counterpart of (1). Under the assumption of weak inhomogeneity, linear solutions are approximated at leading order by waves of the form $\exp \left((i / \epsilon) \int^{X} k(u) d u-i \omega t\right)$. The corresponding local linear dispersion relation reads

$$
\omega=\Omega^{l}(k, X) \equiv \omega_{0}(X)+\frac{1}{2} \omega_{k k}(X)\left(k-k_{0}(X)\right)^{2},
$$

with associated complex local linear spatial branches

$$
k^{l \pm}(X ; \omega) \equiv k_{0}(X) \pm \sqrt{2 \frac{\omega-\omega_{0}(X)}{\omega_{k k}(X)}} .
$$


As defined in Ref. 5, the complex absolute frequency $\omega_{0}$ necessarily coincides with the branch point of (3): at $\omega=\omega_{0}$ both wave number branches are equal to the local absolute wave number $k_{0}$. The following basic flow structure is assumed: a central absolutely unstable (AU) region of finite extent characterized by $\omega_{0, i}(X) \equiv \operatorname{Im} \omega_{0}(X)>0$, surrounded by convectively unstable (CU) regions with $\omega_{0, i}(X)<0$, which in turn become stable far downstream $(X \rightarrow+\infty)$ and upstream $(X \rightarrow-\infty)$. In order to enforce causality, sufficiently large wave numbers are assumed to be damped, i.e., $\omega_{k k, i}(X)<0$ for all $X$. We assume a basic flow advection towards increasing $X$ which is readily shown to correspond to $k_{0, i}<0$. The + and - superscripts are unambiguously assigned to the spatial branches exponentially decaying towards $X=+\infty$ and $X=-\infty$, respectively.

In unstable regions the CGL equation admits local nonlinear traveling wave solutions of the form $\psi$ $=R(X) \exp \left((i / \epsilon) \int^{X} k(u) d u-i \omega t\right)$, with real wave number $k$, real frequency $\omega$ and real amplitude $R$ governed by the local nonlinear dispersion relation

$$
\omega=\omega_{0}(X)+\frac{1}{2} \omega_{k k}(X)\left(k-k_{0}(X)\right)^{2}+\gamma(X) R^{2} .
$$

This complex equation with three real unknowns can easily be cast in the form

$$
\omega=\Omega^{n l}(k, X)
$$

and

$$
R^{2}=\mathcal{R}_{2}(k, X),
$$

where the functions $\Omega^{n l}$ and $\mathcal{R}_{2}$ are quadratic polynomials in $k$ with $X$-dependent real coefficients. The real nonlinear wave number branches $k^{n l \pm}(X ; \omega)$ are derived from $(5 \mathrm{a})$; the allowed wave numbers are those for which $\mathcal{R}_{2}(k, X)>0$. We only consider CGL coefficients for which the finite amplitude traveling waves are stable.

Global mode solutions over the entire flow are obtained by asymptotically matching together local traveling wave solutions of the same frequency. By definition, a global mode is necessarily made up of the decaying $k^{l-}$ branch for $X \rightarrow$ $-\infty$ and the decaying $k^{l+}$ branch for $X \rightarrow+\infty$. The frequency selection gives rise to a nonlinear eigenvalue problem: the matching of finite amplitude oscillations in a central region to exponentially decaying tails in both upstream and downstream directions can only be achieved for a specific frequency.

This problem has been solved for purely linear global modes in Ref. 6. In the fully nonlinear regime, soft global modes with an overall slowly varying spatial envelope have been identified and described in Ref. 14; their real global frequency $\omega_{s}$ is obtained at a saddle point $\left(k_{s}, X_{s}\right)$ of the nonlinear dispersion relation $\Omega^{n l}(k, X)$. The objective of this letter is to report the existence of a second type of fully nonlinear solutions: in situations where soft modes fail to exist, they are replaced by a steep mode with a sharp front.

The spatial structure of a steep global mode of frequency $\omega_{f}$ is given in Fig. 1(a). Such a solution is characterized by a sharp front at the upstream boundary $X_{f}$ of the AU region indicated in gray. Associated linear $k^{l \pm}$ and nonlinear $k^{n l \pm}$ wave number branches at the frequency $\omega_{f}$ vary along the
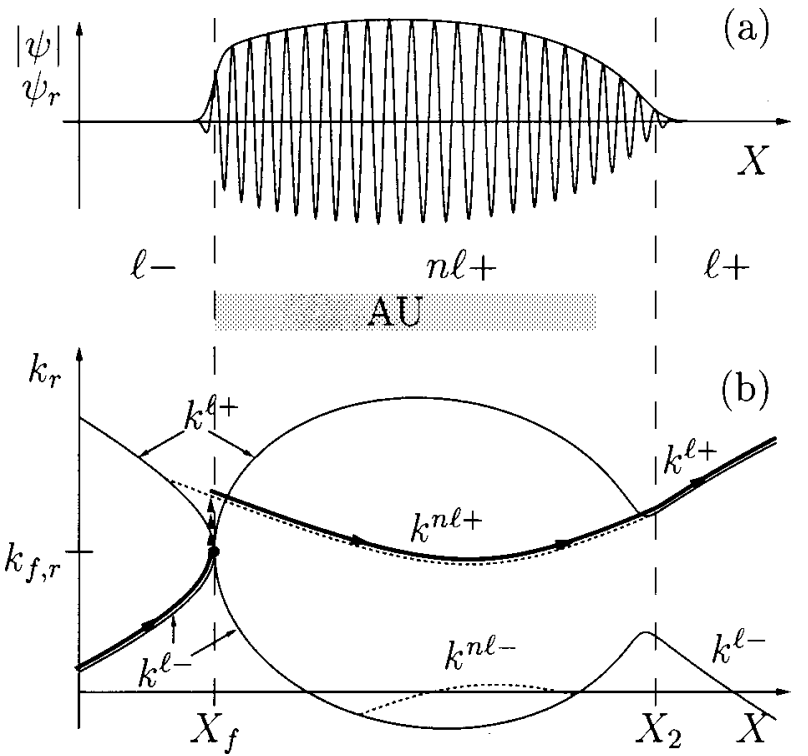

FIG. 1. (a) Envelope $|\psi|$ and real part $\psi_{r}$ of steep global mode with sharp front at upstream boundary $X_{f}$ of $\mathrm{AU}$ region (in gray). (b) Corresponding linear and nonlinear spatial branches in the $X-k_{r}$ plane. Local wave number making up solution in (a) follows path indicated by a thick line. The wave number jump at the front is indicated by repeated arrows.

streamwise direction $X$, as shown by solid and dashed lines in the $X-k_{r}$ plane of Fig. 1(b). The local wave number making up the actual solution follows the path indicated by a thick line in Fig. 1(b). Except for the jump at the front [repeated arrows in Fig. 1(b)] local wave number and amplitude vary slowly. The nonlinear solution prevails in the region $X_{f}<X<X_{2}$ which extends beyond the AU region.

The steep global frequency $\omega_{f}$ and the front location $X_{f}$ are then solely determined by applying the following criterion to the local linear dispersion relation:

$$
\omega_{f}=\Omega^{l}\left(k_{f}, X_{f}\right),
$$

where the pair $\left(k_{f}, X_{f}\right)$ satisfies

$$
\frac{\partial \Omega^{l}}{\partial k}\left(k_{f}, X_{f}\right)=0 \quad \text { and } \Omega_{i}^{l}\left(k_{f}, X_{f}\right)=0 .
$$

In terms of $\omega_{0}(X)$ these conditions read

$$
\omega_{f}=\omega_{0}\left(X_{f}\right) \text { and } \omega_{0, i}\left(X_{f}\right)=0 .
$$

The above equations are reminiscent of the Dee-Langer selection criterion ${ }^{15}$ for a propagating front connecting an unstable state $\psi=0$ to a fully nonlinear wave pattern in a homogeneous medium undergoing a supercritical bifurcation. According to this criterion, a stationary front exists in homogeneous media only when the control parameters are chosen to be exactly at the CU/AU transition. In the present inhomogeneous, i.e., spatially varying context, the CU/AU transition precisely occurs at the single location $X_{f}$. The front at this station has the overall frequency $\omega_{f}$ and the complex wave number at the decaying front edge coincides [Fig. 1(b)] with the local absolute wave number $k_{f}=k_{0}\left(X_{f}\right)$ $=k^{l \pm}\left(X_{f}, \omega_{f}\right)$.

By definition of the absolute wave number, the linear $k^{l \pm}$ branches meet at $k_{f}$ for $\omega=\omega_{f}$ and $X=X_{f}$. In a neighborhood of $X_{f}$, the upstream $k^{l-}$ branch decays towards $X$ 
$=-\infty$ when $X<X_{f}$, whereas the downstream spatially amplified $k^{l+}$ branch is replaced by the nonlinear $k^{n l+}$ branch when $X>X_{f}$. The front at $X_{f}$ exactly connects the linear $k^{l-}$ branch in the $\mathrm{CU}$ region $X<X_{f}$ with the nonlinear $k^{n \ell+}$ branch prevailing in the AU region $X>X_{f}$. Further downstream the nonlinear $k^{n l+}$ branch eventually returns to the linear $k^{l+}$ via a neutral stability station $X_{2}$ where the nonlinear amplitude vanishes, exactly as for soft global modes. ${ }^{14}$

A front located at the upstream boundary of the AU region is a stable configuration due to the following argument. Consider a small displacement from the front equilibrium position at $X_{f}$ towards $X>X_{f}$. The front now experiences a slightly AU medium and hence ${ }^{15}$ propagates slowly towards its decaying edge, i.e., upstream. When the front is displaced to $X<X_{f}$, it penetrates into a $\mathrm{CU}$ region and is thus pushed downstream. In any case the front is seen to return to its equilibrium position. The complete asymptotic representation of steep global modes is obtained by linear and nonlinear WKBJ matching techniques as in Ref. 14.

According to the present theory the CGL equation with spatially varying coefficients has been shown to admit two types of time harmonic solutions. Soft global modes are characterized by a nonlinear saddle point condition, ${ }^{14}$ whereas steep global modes display a stationary front (6) and (7) at the upstream boundary of the AU domain. The nature of the selected global mode is determined by formally computing the respective characteristic frequencies $\omega_{s}$ and $\omega_{f}$ : in a future publication we will show that the mode of largest frequency is selected and that no other global mode type occurs. The validity of these theoretical criteria is confirmed by spatio-temporal numerical simulations of (1). Furthermore, according to the results of Ref. 16, one expects the nature of the bifurcation to a fully nonlinear global mode to be extremely sensitive to $\epsilon$.

In both instances, frequency selection takes place at the downstream position where a - branch is linked to a + branch: $k^{n l-}$ and $k^{n l+}$ at $X_{s}$ for a soft global mode, $k^{l-}$ and $k^{n l+}$ at $X_{f}$ for a steep global mode. These stations effectively act as frequency generators for the entire flow. Such locations may be interpreted as local oscillators inducing the upstream - branch and the downstream + branch, regardless whether these branches are linear or nonlinear. It is noteworthy that the present steep frequency selection criterion (6) and (7) demonstrates, in the CGL context, the validity of the initial resonance principle postulated by Monkewitz and Nguyen ${ }^{17}$ to account for self-excited resonances in bluff body wakes.

An essential difference between steep and soft global modes is that steep global modes only involve one nonlinear spatial branch $k^{n l+}$. The sharp front allows an immediate crossover from the linear - to the nonlinear + branch. Generalization of the present theory to real flows is in progress: In the context of free shear flows governed by the NavierStokes equations, the local $k^{n l+}$ is represented by fully nonlinear saturated solutions on a streamwise periodic domain as obtained in direct numerical simulations for a given parallel basic flow. To our knowledge local $k^{n l-}$ branches for free shear flows have never been identified. It therefore seems likely that fully nonlinear global modes in wakes, jets or shear-layers may be described as steep rather than soft global modes.

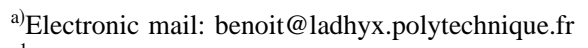

${ }^{1} \mathrm{P}$. Huerre and P. Monkewitz, "Absolute and convective instabilities in free shear layers,", J. Fluid Mech. 159, 151 (1985).

${ }^{2} \mathrm{~W}$. Koch, "Local instability characteristics and frequency determination on self-excited wake flows," J. Sound Vib. 99, 53 (1985).

${ }^{3} \mathrm{P}$. A. Monkewitz, "The absolute and convective nature of instability in two-dimensional wakes at low Reynolds numbers,' Phys. Fluids 31, 999 (1988).

${ }^{4}$ P. A. Monkewitz and K. D. Sohn, "Absolute instability in hot jets," AIAA J. 26, 911 (1988).

${ }^{5}$ A. Bers, in Handbook of Plasma Physics, edited by M. N. Rosenbluth and R. Z. Sagdeev (North-Holland, Amsterdam, 1983).

${ }^{6}$ J.-M. Chomaz, P. Huerre, and L. G. Redekopp, "A frequency selection criterion in spatially developing flows," Stud. Appl. Math. 84, 119 (1991).

${ }^{7}$ P. A. Monkewitz, P. Huerre, and J.-M. Chomaz, "Global linear stability analysis of weakly non-parallel shear flows," J. Fluid Mech. 251, 1 (1993).

${ }^{8}$ D. A. Hammond and L. G. Redekopp, "Global dynamics and aerodynamics flow vectoring of wakes," J. Fluid Mech. 338, 231 (1997).

${ }^{9}$ J.-M. Chomaz, "Absolute and convective instabilities in nonlinear systems,"’ Phys. Rev. Lett. 69, 1931 (1992).

${ }^{10}$ A. Couairon and J.-M. Chomaz, "Absolute and convective instabilities, front velocities and global modes in nonlinear systems," Physica D 108, 236 (1997).

${ }^{11}$ N. Meunier, M. R. E. Proctor, D. D. Sokoloff, A. M. Soward, and S. M. Tobias, "Asymptotic properties of a nonlinear $\alpha \omega$-dynamo wave: period, amplitude and latitude dependence," Geophys. Astrophys. Fluid Dyn. 86, 249 (1997).

${ }^{12}$ P. Büchel, M. Lücke, D. Roth, and R. Schmitz, "Pattern selection in the absolutely unstable regime as a nonlinear eigenvalue problem: Taylor vortices in axial flow,', Phys. Rev. E 53, 4764 (1996).

${ }^{13}$ H. W. Müller, M. Lücke, and M. Kamps, "Transversal convection patterns in horizontal shear flow," Phys. Rev. A 45, 3714 (1992).

${ }^{14} \mathrm{~B}$. Pier and P. Huerre, "Fully nonlinear global modes in spatially developing media," Physica D 97, 206 (1996).

${ }^{15}$ G. Dee and J. S. Langer, “Propagating pattern selection," Phys. Rev. Lett. 50, 383 (1985).

${ }^{16}$ S. Le Dizès, P. Huerre, J.-M. Chomaz, and P. A. Monkewitz, Proceedings of the IUTAM Symposium on Bluff-Body Wakes, Dynamics and Instabilities, edited by H. Eckelmann et al. (Springer, Berlin, 1993), p. 147.

${ }^{17} \mathrm{P}$. A. Monkewitz and L. N. Nguyen, "Absolute instability in the nearwake of two-dimensional bluff bodies," J. Fluids Struct. 1, 165 (1987). 